\title{
Monophosphoryl Lipid A
}

National Cancer Institute

\section{Source}

National Cancer Institute. Monophosphoryl Lipid A. NCI Thesaurus. Code C87754.

A modified form of lipid A, the biologically active part of Gram-negative bacterial lipopolysaccharide (LPS) endotoxin, and a Toll-like receptor 4 (TLR4) agonist, with potential immunostimulatory activity. As a vaccine adjuvant, monophosphoryl lipid A (MPLA) stimulates both cellular and humoral responses to the vaccine antigen. Compared to LPS, MPLA exerts a similar immunostimulatory activity but with reduced toxicity. 